\title{
Assessing the impact of relaxing cruise operations with a reduction of the minimum rate of climb and/or step climb heights
}

\author{
Ramon Dalmau ${ }^{\mathrm{a}}$, Xavier Prats ${ }^{\mathrm{a}, *}$ \\ ${ }^{a}$ Telecommunications and Aerospace Engineering School of Castelldefels, Technical \\ University of Catalonia Barcelona Tech., Catalonia, Spain
}

\begin{abstract}
A compromise solution to increase flight efficiency in cruise, but without penalising capacity (or even safety), would be perhaps to remove (or relax) the minimum rate of climb (ROC) constraint and/or to reduce the height of the step climbs in cruise. In this paper, the benefits (in terms of total operating costs) and the associated impact on the air traffic management (ATM) of such "relaxed cruise" operations are quantified for a representative mediumhaul aircraft under different scenarios, by means of an in-house trajectory optimisation software. Results show that by reducing the minimum ROC from 500 to $300 \mathrm{ft} \mathrm{min}^{-1}$, whilst keeping the step climb height according to current reduced vertical separation minima (RVSM) standard would give a good compromise between cost savings and impact on the ATM.
\end{abstract}

Keywords: relaxed cruise operations, continuous cruise climb, rate of climb, step climb height

\section{Introduction}

An optimal vertical flight profile for a turbojet aircraft, in terms of flight efficiency, consists of a continuous climb up to reaching an optimal altitude and speed. Then, as long as the weight of the aircraft decreases (due to fuel burnt), climb continues at a much lower rate of climb (ROC) until a continuous descent at idle thrust can be initiated $[1,2,3,4]$. In previous works [5, 3],

\footnotetext{
${ }^{*}$ Corresponding author. Tel.: +34 934134126

E-mail addresses: ramon.dalmau@upc.edu (R. Dalmau), xavier.prats@upc.edu (X. Prats)
} 
the authors computed the benefits of such continuous cruise climbs for maximum range operations (i.e. only fuel was minimised). Results showed a reduction in fuel consumption (only for the cruise phase) ranging from $0.5 \%$ to $2 \%$, with respect to optimal conventional operations. Interestingly, trip time was also decreased when flying such continuous optimal profiles between $1 \%$ and $5 \%$. With the same aim, in [6] continuous cruise climbs were compared with real flight data collected during one day of domestic US operations. In this case, results showed that a fuel burn reduction up to $3.5 \%$ is possible in cruise given complete freedom to optimise altitude and speed.

At present, however, flying at constant cruise altitudes (flight levels) is one of the key elements to strategically separate flows of aircraft. The benefits of continuous cruise climbs have only been shown in demonstration flight trials, such as reported in $[7,8]$. According to [9], continuous cruise operations could be implemented in a relatively short time-frame with current aircraft types, requiring changes to existing procedures and published routes, but not needing extra equipment on-board. Nevertheless, unless a disrupting change in the air traffic managment (ATM) systems and procedures is produced, a massive implementation of continuous cruise climbs in dense airspaces will not be a reality in the near future. This could be inferred by the preliminary results of a recent work that shows how such continuous cruise climbs would increase traffic complexity in the European airspace [10], detecting four times more conflicts than in conventional operations.

Historically, standard Vertical Separation Minima (VSM) between two consecutive flight levels was 1,000 ft from the surface to FL290; and 2,000 ft above FL290. Aiming to theoretically double airspace capacity and trying to allow aircraft to operate closer to their optimum altitude, in 1982 the Reduced VSM (RVSM) program was initiated by the International Civil Aviation Organization (ICAO). The VSM was reduced from 2, $000 \mathrm{ft}$ to 1, $000 \mathrm{ft}$. Nowadays, RVSM is implemented almost world-wide up to FL410 [11]. Besides vertical separation standards, most countries apply flight level orientation schemes, meaning that eastbound and westbound traffic flows are allocated, respectively, on odd and even flight levels [12].

With current concept of operations, and when the route is long enough, aircraft change their cruise level one or several times during the flight (the socalled step climbs). In this way, they intend to fly as close as possible to their optimal (and continuous) vertical profile. A step climb is always subject to an air traffic control (ATC) clearance and the exact location along the route depends on variables such as the aircraft mass, the weather conditions and 
the spacing between two consecutive flight levels (i.e. the height of the step climb). In order to ease the prediction of flight profiles when performing separation tasks, ATC also imposes a minimum ROC to perform any step climb. According to the Federal Aviation Administration (FAA) [13], and most European regulations (see for instance [14]), a minimum ROC of $500 \mathrm{ft} \mathrm{min}{ }^{-1}$ is enforced to all aircraft. This constraint is typically applied for the whole trajectory and becomes very important for ATC in terminal airspace, where traffic is either climbing or descending. Nevertheless, this bound is especially relevant for the aircraft operator when planning step climbs, since it significantly limits the capability to climb to a higher flight level due to the relatively low excess power available at typical cruise altitudes.

A compromise solution to increase flight efficiency in cruise, without penalising capacity (or even safety), would be perhaps to allow shallower climbs between two consecutive flight levels (i.e. removing or relaxing the minimum ROC constraint) and/or to reduce the height of the step climbs (i.e. removing or locally adapting the flight level orientation scheme). Furthermore, these "relaxed cruise" operations could eventually be used as a first step towards a full deployment of continuous cruise climb in the future.

In this paper, the potential benefits (in terms of total operating costs) of such relaxed cruise operations are investigated for different ROC minima limitations and heights of the step climbs. In addition, the impact on the ATM of the proposed operations is analysed by means of two different metrics: the number of cruise level changes per nautical mile and the percentage of the total trip distance that is not flown at a constant altitude.

The assessment is performed for a representative medium-haul aircraft, by means of an in-house trajectory optimisation software [5, 3]. This software uses accurate aircraft performance data from the manufacturer, providing in this way, more realistic fuel consumption and flight time figures than other optimisation frameworks based on the Eurocontrol's Base of Aircraft Data (BADA) version 3.x, which has been reported to be not accurate enough to compute optimal cruise flight profiles [15].

Section 2 presents the experimental setup, including the methodology to compute optimal trajectories for the different types of operations and the different scenarios investigated herein. In Sections 3 and 4, the effects of the reduction of the minimum $\mathrm{ROC}$ and the height of the step climbs are presented, respectively. Finally, the conclusions are addressed in Section 5. 


\section{Experimental setup}

The potential benefits (and associated impact on the ATM) of relaxed cruise operations have been investigated for an Airbus A320, a typical twoengine, narrow-body, commercial transport aircraft. Accurate aircraft performance data, derived from Airbus Performance Engineering Programs (PEP), have been used to model drag, engine thrust and fuel flow aiming to obtain representative optimal trajectories.

In Section 2.1 the methodology to compute these optimal trajectories is addressed. The selected scenarios are presented in Section 2.2.

\subsection{Trajectory computation}

The optimisation of an aircraft trajectory can be formulated as a multiphase constrained optimal control problem [16]. In this kind of problems, the aircraft is represented as a system described by a state vector $\boldsymbol{x}$ and a control vector $\boldsymbol{u}$. In this paper, $\boldsymbol{x}$ is composed by true airspeed, the mass of the aircraft, the altitude and distance to go; and $\boldsymbol{u}$ is composed by the thrust of the engines and the aerodynamic flight path angle.

The goal of the optimal control problem is to find the time history of $\boldsymbol{u}$ and optional time-independent parameters $\boldsymbol{p}$ that minimise a given cost function $J$ (such as trip fuel and/or time), defined over an either free or fixed interval of time $\left[t_{0}, t_{f}\right]$. In order to guarantee a representative and operationally feasible trajectory, as a result of the optimisation process, several constraints must be considered. In particular, the dynamics of the system $\boldsymbol{f}$ expressed by non-linear vector functions:

$$
\frac{\mathrm{d} \boldsymbol{x}}{\mathrm{d} t}=\dot{\boldsymbol{x}}(t)=\boldsymbol{f}(\boldsymbol{x}(t), \boldsymbol{u}(t), \boldsymbol{p}, t)
$$

In this paper, a point-mass representation of the aircraft (where forces are applied at its centre of gravity) reduced to what is commonly called a "gamma-command" model has been used. Further details of the equations describing this model can be found in [5].

In addition, $\boldsymbol{x}$ is typically fixed to some initial conditions:

$$
\boldsymbol{x}\left(t_{0}\right)=\boldsymbol{x}_{0}
$$

and the solution might also satisfy some terminal constraints:

$$
\boldsymbol{\psi}\left(\boldsymbol{x}\left(t_{f}\right), t_{f}\right)=0
$$


algebraic path constraints:

$$
\boldsymbol{g}(\boldsymbol{x}(t), \boldsymbol{u}(t), \boldsymbol{p}, t) \leq 0
$$

modelling the aircraft envelope, ATM restrictions and aircraft operational procedures; and box constraints (also known as bounds) on the state, control and parameter variables:

$$
\begin{aligned}
\boldsymbol{x}_{L}(t) & \leq \boldsymbol{x}(t) \leq \boldsymbol{x}_{U}(t) \\
\boldsymbol{u}_{L}(t) & \leq \boldsymbol{u}(t) \leq \boldsymbol{u}_{U}(t) \\
\boldsymbol{p}_{L} & \leq \boldsymbol{p} \leq \boldsymbol{p}_{U}
\end{aligned}
$$

In the previous notation, $(\cdot)_{L}$ and $(\cdot)_{U}$ are respectively the lower and upper bounds of these box constraints.

For those problems defined over more than one phase, the dynamics of the system, the terminal, path and box constraints might be different in each phase. However, it might be desirable to link the state variables across two consecutive phases. This is accomplished by enforcing link constraints.

There are several ways to solve the optimal control problem stated above. In this paper direct collocation methods have been used. Such direct methods transform the original continuous (and thus infinite) optimal control problem into a (discrete and finite) Non-Linear Programming (NLP) problem[17, 18].

The time histories of control and state variables are discretised at a set of collocation points, being the differential equations (1) approximated by some continuous function (such as polynomials) over each collocation step. The values of these discretised variables, along with $\boldsymbol{p}$ become the unknowns of the new finite variable problem, which can be formulated as a NLP problem and solved by NLP solvers [5, 3].

In this paper, the optimisation problem has been solved by using solvers CONOPT (as NLP) and SBB as MINLP (mixed integer non-linear programming), both bundled into the GAMS ${ }^{1}$ software suite.

It should be noted that some of the constraints (1)-(5) are independent of the type of operation being modelled (such as continuous cruise climb or conventional cruise operations at constant altitude). For instance, the dynamics of the aircraft or generic bounds on the state and control variables

\footnotetext{
${ }^{1}$ www.gams.de
} 
(such as maximum and minimum operating speeds or flight path angles). The remaining constraints of the problem depend on the particular type of operation being modelled, and are specified by means of a flight profile. The flight profile is divided in several phases, where various path, terminal or box constraints may apply in order to represent a given type of operation.

The formulation of the optimal control problem for the optimisation of continuous cruise climb and conventional operations has been addressed in previous publications $[5,3]$. Next, the flight profile for the types of operation considered herein are presented for the sake of completeness.

\subsubsection{Computation of continuous cruise climb}

For the optimisation of continuous cruise climb, the flight profile is composed by just one phase and ATM constraints are not considered (such as minimum ROC, specific ATM speed limits or the requirement to fly at constant cruise altitudes). In this type of operation, only flight envelope constraints are imposed: maximum operational speeds MMO (maximum Mach in operations) and VMO (maximum velocity in operations) have been set to 0.80 and $340 \mathrm{kt}$, respectively[19]; minimum and maximum flight path angles have been bounded to operationally realistic values; the upper and lower bounds on thrust have been set, respectively, to maximum thrust climb $T_{\max }$ and thrust idle $T_{i d l e}$ (which are functions of the altitude and Mach).

\subsubsection{Computation of conventional operations}

Besides the flight envelope constraints enumerated above, the optimisation of conventional operations requires additional constraints reflecting typical ATM practices and operational procedures. Table 1 summarises all the phases and operational constraints needed to model the flight profile for this type of operation. The mathematical details of how the different constraints have been modelled can be found in [5].

The climb segment is divided in several phases with different models and standard operational procedures, such as constant callibrated airspeed (CAS) or Mach phases. For the cruise of conventional operations, step climbs of 2,000 ft (for RVSM airspace) or 4,000 ft (for standard VSM airspace) in between cruise levels are modelled and, without loss of generality, odd flight levels are considered in this paper. Cruise phases are assumed to be at constant Mach and altitude and one minute of acceleration/deceleration is allowed at the beginning of each one of them to adjust the cruise speed. 
Table 1: Definition of the flight phases and their operational constraints for conventional operations

\begin{tabular}{|c|c|c|}
\hline Phase \# & Description & Remarks \\
\hline 1 & Take-off acceleration & Constant acceleration \\
\hline 2 & Constant CAS climb & CAS $<250$ kt under FL100 \\
\hline 3 & Climb acceleration & Constant acceleration above FL100 \\
\hline 4 & Constant CAS climb & \\
\hline 5 & Constant Mach climb & \\
\hline 6 & Cruise speed adjustment & $\begin{array}{c}\text { Adjust speed in less than } 1 \mathrm{~min} \\
\text { Constant altitude }\end{array}$ \\
\hline 7 & Cruise & $\begin{array}{c}\text { Constant Mach } \\
\text { Constant altitude } \\
\text { Cruise time greater than } 5 \text { min }\end{array}$ \\
\hline 8 & Step climb & $\begin{array}{l}\text { Constant Mach } \\
\text { Minimum rate of climb of } \mathrm{ROC}_{\text {min }}\end{array}$ \\
\hline 9 & Cruise speed adjustment & Same as phase \#6 \\
\hline 10 & Cruise & Same as phase \#7 \\
\hline 11 & Step climb & Same as phase \#8 \\
\hline ... & $\ldots$ & $\ldots$ \\
\hline$N-6$ & Cruise & Same as phase \#7 \\
\hline$N-5$ & End of cruise speed adjustment & Same as phase \#6 \\
\hline$N-4$ & Constant Mach descent & \\
\hline$N-3$ & Constant CAS descent & \\
\hline$N-2$ & Descent deceleration & Constant deceleration above FL100 \\
\hline$N-1$ & Constant CAS descent & CAS $<250$ kt under FL 100 \\
\hline$N$ & Approach deceleration & Constant deceleration \\
\hline
\end{tabular}


State-of-the-art flight management systems (FMS) typically impose a minimum cruise distance and/or cruise time when computing a trajectory, preventing in this way too short cruises. These distances/times are in general aircraft type dependent and may be also changed according to airline policy. In this paper, a minimum cruise time of 5 min have been taken into account when computing conventional trajectories. At the end of the last cruise phase

a speed adjustment phase (still at constant altitude) is introduced allowing the aircraft to transition from the best cruise speed to the best descent one.

Finally, the descent segment, which is assumed to be continuous and at idle thrust, is also divided in several phases with standard operational procedures similar to those of the climb.

It should be noted that the optimisation algorithm will choose the (optimal) values of the different CAS, Mach, acceleration/deceleration and altitude phase dependent parameters described above, as well as the number of step climbs (if any) to perform.

Although not explicitly shown in Table 1 for the sake of simplicity, a minimum rate of climb/descent $\mathrm{ROC}_{\text {min }}$ is enforced during the whole climb and descent (besides the step climb phases). Based on current regulations, $\mathrm{ROC}_{\text {min }}$ is set to $500 \mathrm{ft} \min ^{-1}$ for conventional operations $[14,13]$.

\subsubsection{Computation of relaxed cruise operations}

The formulation of the problem for relaxed cruise operations is analogous to that presented in Section 2.1.2 for conventional operations and summarised in Table 1. In this type of operation, however, the height of the step climbs and/or the $\mathrm{ROC}_{\min }$ values are reduced aiming to achieve trajectories as close as possible to the continuous vertical profiles presented in Section 2.1.1. The exact values of step climb height and $\mathrm{ROC}_{\text {min }}$ are subject of study in this paper and the trajectory sensitivity to these variables are assessed by means of different simulations.

\subsection{Simulated scenarios}

The optimal trajectories for the three types of operation presented in Section 2.1 have been computed for several scenarios, as a result of combining five relevant independent variables. These independent variables include trip distance, airline preferences such as the landing mass (LM) and the cost 
index $(\mathrm{CI})^{2}$, and ATM related restrictions (in particular, the $\mathrm{ROC}_{\text {min }}$ and the height of the step climbs). Table 2 shows the range of values considered for each one of these independent variables and for each type of operation.

Table 2: Independent variables considered to generate the different scenarios

\begin{tabular}{|c|c|c|c|}
\hline Variable Operation & Continuous cruise climb & Conventional & Relaxed cruise \\
\hline LM $[\%$ of MLM*] & \multicolumn{3}{|c|}{80,90 and 100} \\
\hline $\mathrm{CI}\left[\mathrm{kg} \min ^{-1}\right]$ & \multicolumn{3}{|c|}{0 and 45} \\
\hline Trip distance $[\mathrm{NM}]$ & \multicolumn{3}{|c|}{400 to 3,000 in increments of 200} \\
\hline $\mathrm{ROC}_{\min }\left[\mathrm{ft} \mathrm{min}^{-1}\right]$ & - & 500 & $100,200,300,400$ \\
\hline Step climbs height $[\mathrm{ft}]$ & - & 2,000 and 4,000 & 2,000 and $1,000^{\dagger}$ \\
\hline
\end{tabular}

* Maximum landing mass

† For these cases only simulations with $\mathrm{ROC}_{\min }$ of 300 and $500 \mathrm{ft} \mathrm{min}^{-1}$ have been performed as justified in light of the obtained results (see next sections).

It should be noted that the scale of the CI depends on the FMS installed on-board. In this paper, the CI is scaled between 0 and 99 .

The two CIs of the experiment have been selected based on the extremes of the range typically used by airlines. According to [21], the traditional speed during cruise is that of Long Range Cruise (LRC). This speed speed is closely related to the Maximum Range Cruise (MRC) speed, which provides the furthest distance traveled for a given amount of fuel burned, thus corresponding to CI 0. The LRC speed is defined as the speed above MRC that results in a $1 \%$ decrease in fuel mileage in terms of distance per fuel burned. The advantage of flying at LRC is that this slightly speed increase provides a noticeable reduction of flight time in exchange for not such a detrimental effect on fuel burn. Nowadays, LRC speed is almost universally higher than the speed that would result from using the CI selected by most carriers.

According to [20], for the Airbus A320, the CI corresponding to LRC speed is around $30 \mathrm{~kg} \mathrm{~min}^{-1}$, depending on the flight level and the gross weight. In this paper, a sightly higher CI has been selected as a representative value of high CI, aiming at contemplating eventual scenarios in which the

\footnotetext{
${ }^{2}$ The CI reflects the relative importance of the cost of time with respect to fuel costs. Therefore, the higher the CI is, the more importance will be given to the trip time and faster optimal aircraft speeds will result from the optimisation process [20]
} 
cost of time is significant if compared to fuel costs.

Relaxed cruise operations have been first simulated by reducing the $\mathrm{ROC}_{\text {min }}$ from $400 \mathrm{ft} \mathrm{min}^{-1}$ to $100 \mathrm{ft} \mathrm{min}^{-1}$ and taking into consideration the current RVSM airspace. Then, relaxed cruise operations with 1,000 ft separation between consecutive cruise levels have been compared with conventional operations under the standard VSM and RVSM. In this case, the $\mathrm{ROC}_{\min }$ in force $\left(500 \mathrm{ft} \mathrm{min}^{-1}\right)$ and a relaxed $\mathrm{ROC}_{\text {min }}$ of $300 \mathrm{ft} \mathrm{min}^{-1}$ have been used. The latter has demonstrated to provide an interesting compromise between cost benefits and impact on the ATM (see next sections).

Next section assesses the benefits (in terms of total operating costs) of the $\mathrm{ROC}_{\text {min }}$ reduction and the consequent impact on the ATM. The analogous study is done in Section 4 for the reduction of the step climbs height.

\section{Effects of the minimum rate of climb restriction}

The climb performance of an aircraft depends on the excess power, which is the difference between the available power $\left(P_{a}=T_{\max } v\right)$ and the required power $\left(P_{r}=D v\right)$; where $v$ and $D$ are, respectively aircraft true airspeed and aerodynamic Drag. As the altitude of the aircraft increases, the density of the air decreases. At first, it may be thought that this decrease in the air density would result in a reduction of $D$. However, in order to maintain the optimal cruise speed and a constant (or slowly changing) altitude, the angle of attack must increase to compensate the loss of lift (due to a loss of air density). The overall impact of these two counteracting effects makes little change in $D$ and, consequently, in the $P_{r}$. Yet, for a turbojet aircraft $P_{a}$ decreases with altitude and since $P_{r}$ is almost constant with altitude, the excess power (and therefore the climb performance) progressively degrades as the aircraft flies to higher altitudes whilst maintaining the optimal cruise speed. As a result, there is an altitude where the excess power is not enough to climb at the $\mathrm{ROC}_{\text {min }}$ enforced by operational constraints.

Typically, aircraft operating ceiling is very close to the optimal altitude and in the majority of situations aircraft are not able to achieve the best cruise performance due to the $\mathrm{ROC}_{\min }$ limitation. The main hypothesis of this paper is that if a less restrictive $\mathrm{ROC}_{\min }$ were allowed, aircraft would be able to reach altitudes closer to the optimal profile, improving the cruise flight efficiency.

To illustrate the effects of the $\mathrm{ROC}_{\min }$ limitation, Fig. 1 shows the vertical profile above FL300, as a function of the distance to go, for different 
$\mathrm{ROC}_{\text {min }}$, gradually increasing from continuous cruise climb (no $\mathrm{ROC}_{\min }$ constraint) to conventional operations $\left(\mathrm{ROC}_{\min }=500 \mathrm{ft} \mathrm{min}{ }^{-1}\right.$, as enforced in current ATM). Respectively, Figs. 1(a) and 1(b) correspond to the scenarios computed with CI 0 and $45 \mathrm{~kg} \mathrm{~min}{ }^{-1}$. In both cases, a trip distance of 2800 NM and a LM of $80 \%$ of the MLM have been considered, and the height of the step climbs has been set to 2,000 ft (RVSM with flight level orientation scheme).

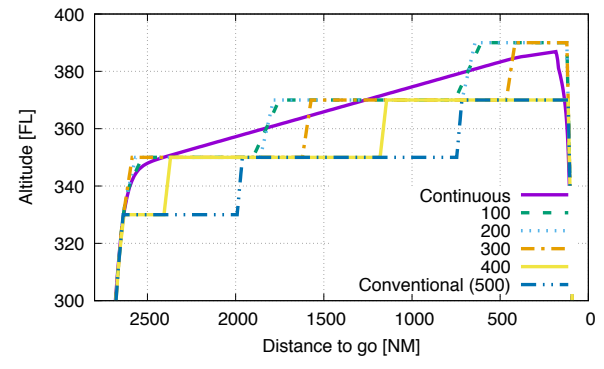

(a) Maximum range

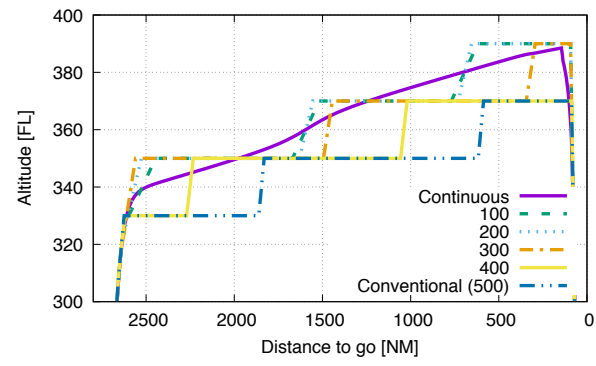

(b) CI $45 \mathrm{~kg} \min ^{-1}$

Figure 1: Vertical profiles for different minimum rates of climb

The unconstrained continuous trajectory follow the so called continuous cruise climb[3], i.e. an uninterrupted climb up to the Top of Descent (TOD), where the continuous descent is initiated. Conversely, for conventional and relaxed cruise operations, the cruise is performed at constant flight levels and step climbs are performed when the excess power allows to climb at $\mathrm{ROC}_{\text {min }}$ towards the next flight level (provided that cruising at this new altitude is more optimal).

For the most restrictive $\mathrm{ROC}_{\text {min }}$, conventional and relaxed cruise operations are not able to climb up to the optimal altitude without violating the minimum ROC constraint. As a result, aircraft fly below the optimal (and continuous) altitude profile and step climbs are performed at the moment the excess power allows to climb to the next flight level at $\mathrm{ROC}_{\min }$.

As the $\mathrm{ROC}_{\text {min }}$ is reduced, relaxed cruise trajectories are capable to closely "follow" the unconstrained continuous cruise climb trajectory and less differences in terms of total operating cost are observed (see next section for a detailed analysis). Relaxed cruise operations with less restrictive $\mathrm{ROC}_{\min }$ are able to reach the optimal altitude with the available excess power, and step climbs are performed when the altitude difference between the optimal altitude and the current flight level is higher than for the next permitted one 
(which depends on the VSM and the flight level orientation scheme).

The effects of the CI on the vertical profile are evident when comparing Fig. 1(a) with Fig. 1(b): the climb profile becomes shallower and the descent profile turns steeper with increasing CI, "moving" forward the location of the top of climb (TOC), the cruise level changes and the TOD.

In Fig. 1 the three different types of operations investigated herein were compared for a particular landing mass and trip distance. In this paper, the effects of these two independent variables have been analysed by varying their values according to the ranges presented in Table 2. As expected, the number of step climbs increases with the trip distance, and under a certain (short enough) trip distance no step climbs are performed (i.e. the optimiser selects only one cruise level). It has been also observed that the effect of the landing mass is analogous to that of the CI.

\subsection{Impact on the operating costs}

Fig. 2 shows the relative operating cost differences between continuous cruise climb and relaxed cruise operations, as a function of the different independent variables shown in Table 2. The maximum extra cost, which is achieved by flying conventional operations $\left(\mathrm{ROC}_{\min }=500 \mathrm{ft} \mathrm{min}^{-1}\right)$, is also shown in this Figure as the worst case. Note that for maximum range operations $(\mathrm{CI}=0)$ the operating cost is equivalent to the fuel consumption.

According to Fig. 2 the extra cost for conventional operation ranges from $0.5 \%$ to $1.1 \%$. As expected, for relaxed cruise operations the extra cost (ranging from $0.2 \%$ to $0.6 \%$ ) is always lower than for conventional operations, demonstrating the benefits in terms of operating costs that could be achieved by reducing the $\mathrm{ROC}_{\text {min }}$.

For the three types of operations studied in this paper, the fuel consumption increase almost in a linear way with the trip distance. Let $f_{i}=a_{i} d+b_{i}$ $(i \in\{\mathrm{cnt}, \mathrm{cnv}, \mathrm{rlxd}\})$ be the linear functions describing the fuel consumption of continuous cruise climb, conventional, and relaxed operations, respectively, as a function of the trip distance $d$. The relative extra fuel consumption of the type of operation $j \in\{\mathrm{cnv}, \mathrm{rlxd}\}$ with respect to continuous cruise climb can be computed as follows:

$$
f_{j}^{\text {extra }}=100 \frac{f_{j}-f_{\mathrm{cnt}}}{f_{\mathrm{cnt}}}=100 \frac{a_{j}^{\text {extra }} d+b_{j}^{\text {extra }}}{a_{\mathrm{cnt}} d+b_{\mathrm{cnt}}}
$$

where $a_{j}^{\text {extra }}=a_{j}-a_{\text {cnt }}$ and $b_{j}^{\text {extra }}=b_{j}-b_{\text {cnt }}$ are the slope and intercept of 

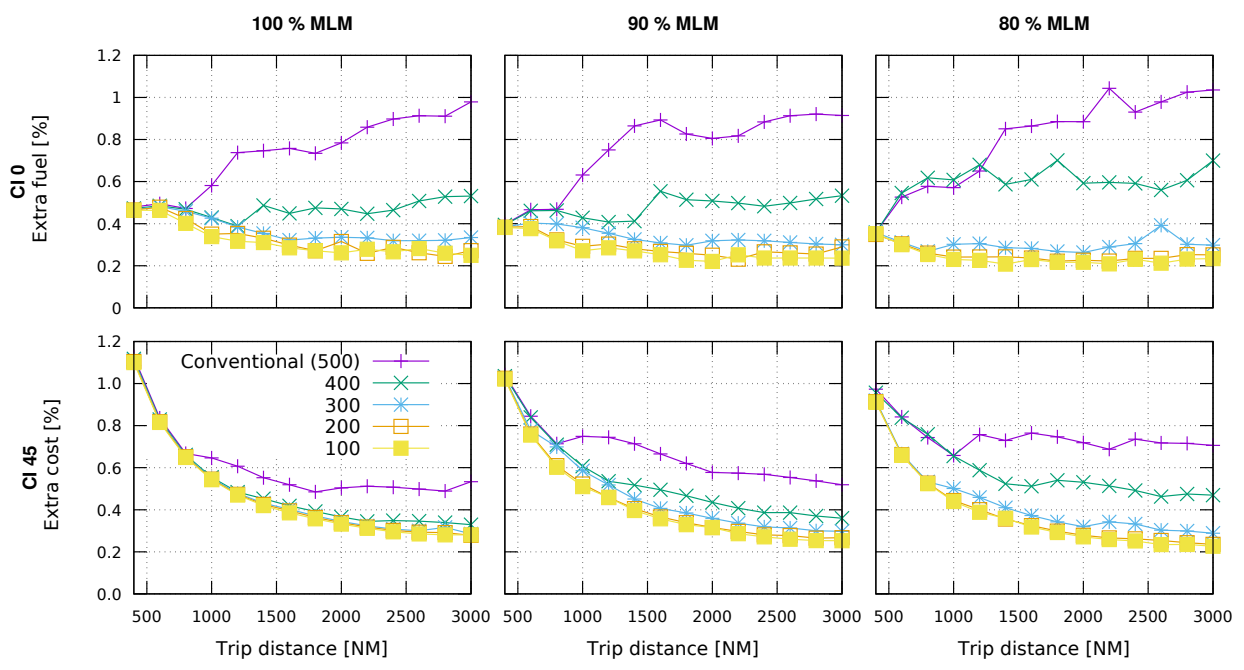

Figure 2: Relative operating cost differences with respect to continuous cruise climb for different minimum rates of climb

the (also linear) function representing the absolute extra fuel consumption of the type of operation $j$ with respect to continuous cruise climb.

As it can be seen from Eq. (6), $\lim _{d \rightarrow 0} f_{j}^{\text {extra }}=b_{j}^{\text {extra }} / b_{\text {cnt }}$ and $\lim _{d \rightarrow \infty} f_{j}^{\text {extra }}=$ $a_{j}^{\text {extra }} / a_{\text {cnt }}$. Therefore, three different behaviours of the relative extra fuel consumption are possible, depending on the relative values of the slopes and intercepts of the concerned linear functions:

1. $\lim _{d \rightarrow 0} f_{j}^{\text {extra }}=\lim _{d \rightarrow \infty} f_{j}^{\text {extra }} \Longrightarrow b_{j}^{\text {extra }} / b_{\text {cnt }}=a_{j}^{\text {extra }} / a_{\text {cnt }}:$ the relative extra fuel consumption is constant for all $d$. This is a very uncommon case, and has not been observed in this study

2. $\lim _{d \rightarrow 0} f_{j}^{\text {extra }}>\lim _{d \rightarrow \infty} f_{j}^{\text {extra }} \Longrightarrow b_{j}^{\text {extra }} / b_{\text {cnt }}>a_{j}^{\text {extra }} / a_{\text {cnt }}$ : the relative extra fuel consumption first decreases with $d$ and then stabilises to a constant value

3. $\lim _{d \rightarrow 0} f_{j}^{\text {extra }}<\lim _{d \rightarrow \infty} f_{j}^{\text {extra }} \Longrightarrow b_{j}^{\text {extra }} / b_{\text {cnt }}<a_{j}^{\text {extra }} / a_{\text {cnt }}$ : the relative extra fuel consumption first increases with $d$ and then stabilises to a constant value

For conventional operations and relaxed cruise operations with $400 \mathrm{ft} \mathrm{min}^{-1}$ the third behaviour discussed above is observed. As explained above, conventional and relaxed cruise operations under very restrictive $\mathrm{ROC}_{\text {min }}$ are not 
able to achieve the optimal altitude due to the low excess power at typical cruise altitudes and, consequently, fly always below the optimal altitude profile (see Fig. 1). This fact implies that the absolute extra fuel consumption with respect to the continuous cruise climb rapidly increases with the trip distance if compared with the total fuel consumption.

Conversely, the second behaviour is observed for lower rates of climb: relaxed cruise operations "cross" the trajectory of the continuous cruise climb, performing step climbs whenever the next permitted flight level is more optimal than the currently selected (provided that the excess power allows to climb at $\left.\mathrm{ROC}_{\text {min }}\right)$. In such case, the absolute extra fuel consumption with respect to continuous cruise climb slowly increases with the trip distance if compared with the total fuel consumption. However, for shorter flights the extra fuel consumption is similar to that obtained with $\mathrm{ROC}_{\min }=500 \mathrm{ft} \mathrm{min}{ }^{-1}$.

For conventional and relaxed cruise operations with CI $45 \mathrm{~kg} \mathrm{~min}^{-1}$, the relative extra cost (which is now a function of fuel consumption and time) always present the second behaviour. This fact is caused by the introduction of the cost of time in the objective function to minimise. While the relative extra fuel consumption follows a similar pattern to that observed for maximum range operations, the absolute extra time increases very slowly with distance (if compared with the total trip time) because both conventional, relaxed cruise and continuous cruise climb select similar cruise speeds (close to the Mach corresponding to the drag rise region).

It is also worth noting that the reduction of the $\mathrm{ROC}_{\text {min }}$ below $300 \mathrm{ft} \mathrm{min}^{-1}$ would not imply a relevant decrease in the total operating cost.

Regarding the LM, no clear pattern can be inferred from Fig. 1 for $\mathrm{ROC}_{\min }$ below $300 \mathrm{ft} \mathrm{min}^{-1}$. Nevertheless, for higher $\mathrm{ROC}_{\min }$ the relative extra cost seems to sightly decrease with increasing LM. In any case, it can be concluded that the dependence of the relative extra cost on this variable is much lower than on the $\mathrm{ROC}_{\min }$.

The discontinuities observed in Fig. 2 are mainly due to the discrete behaviour of the conventional and relaxed cruise operations: since only discrete cruise altitudes are allowed, optimal flight altitudes suddenly change at the moment the next flight level becomes more efficient to the current one. This is the cause of the discontinuities observed on the fuel consumption and flight time, and consequently, the total operating cost. 


\subsection{Impact on the ATM}

Notwithstanding the apparent benefits of relaxed cruise operations with reduced $\mathrm{ROC}_{\text {min }}$ discussed in the preceding Section, the consequent impact on the ATM must be also investigated when assessing the feasibility of a future implementation of such operations. The number of step climbs per nautical mile and the percentage of the total trip distance which is not performed at constant altitude (including climb, descent and step climb phases) have been chosen as metrics to quantify their impact on the ATM.

On the one hand, each change of cruise level requires an ATC clearance, involving an increase in ATC workload. On the other hand, the amount of distance (or time) not at constant altitude should be reduced to ease ATC prediction and separation tasks and therefore not increasing the workload.

Figure 3 displays these two metrics for the different independent variables of Table 2. Each column of the histogram shown in this Figure has been obtained by computing the average climb/descent distances (or average number of cruise level changes) for all trip distances given a same CI, LM, $\mathrm{ROC}_{\min }$ and step climbs height. Note that the percentage of trip distance is calculated with respect to the total trip distance, while the second metric is calculated by taking into account only the total cruise distance.
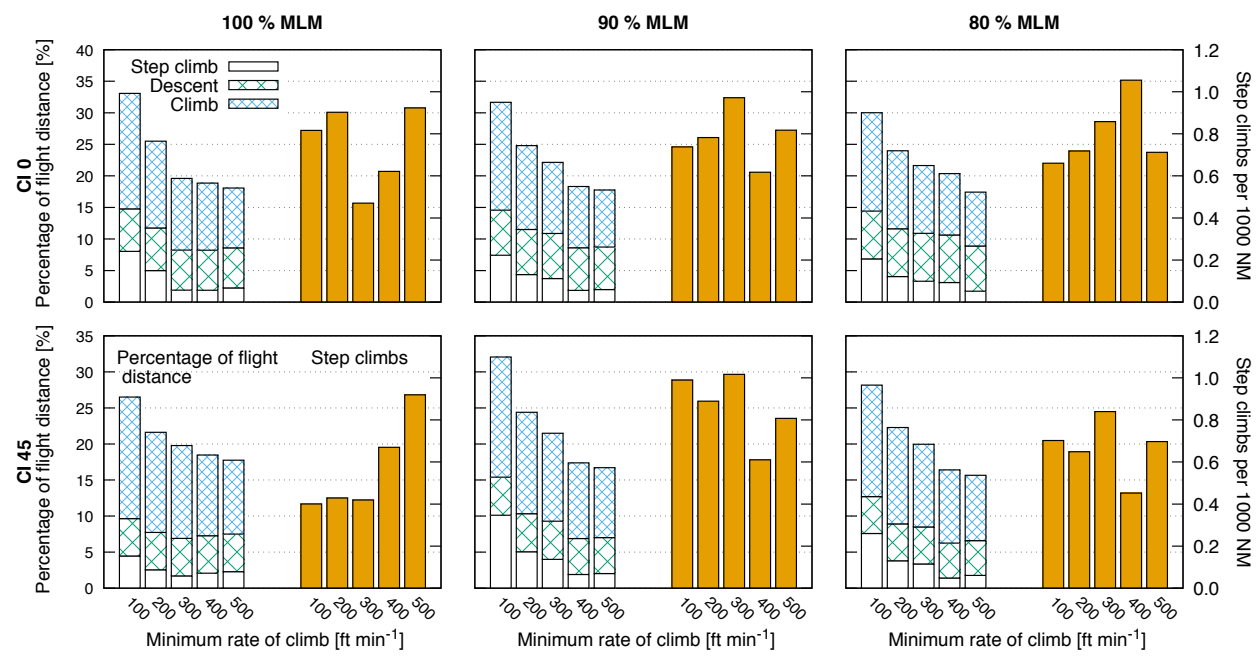

Figure 3: Impact of the ATM as a function for different minimum rates of climb

As expected, as the $\mathrm{ROC}_{\text {min }}$ becomes less restrictive, the percentage of 
cruise distance decreases and more time is spent in the phases not at constant altitude. In general, the altitude of the last and first cruise levels tend to be higher for less restrictive $\mathrm{ROC}_{\text {min }}$. This is why the percentage of distance spent in climb and, into a less extent, descent phases increases as $\mathrm{ROC}_{\min }$ decreases. However, an optimal descent is performed at a rate of descent (ROD) higher than the minimum (in absolute terms) allowed. Instead, the last part of the climb phase is typically performed at $\mathrm{ROC}_{m i n}$, aiming to asymptotically intercept the first cuise altitude. Consequently, the percentage of flight spent in climb increases much more significantly than that in the descent phase when decreasing the $\mathrm{ROC}_{\text {min }}$, as shown in Fig. 3.

It is also interesting to note that the amount of step climbs per nautical mile does not follow a clear pattern. The number of step climbs is a complex function of all the five independent variables considered in this study, due to the discrete behaviour of the step climbs and the high amount of constraints involved in the optimisation problem. In general, for a given trip distance the number of step climbs is almost the same but the initial and final cruise levels are lower for the most restrictive $\mathrm{ROC}_{\text {min }}$ (see for instance Fig. 1).

The higher time used to perform each step climb as a result of reducing $\mathrm{ROC}_{\min }$ combined with the almost unmodified number of step climbs leads to an even more significant increase of the distance not at constant cruise altitude. However, this statement is not always true. For these scenarios with $100 \%$ of MLM the number of step climbs increases with the $\mathrm{ROC}_{\min }$ for 300,400 and $500 \mathrm{ft} \mathrm{min}^{-1}$. Although time is spent in each step climb, the overall result of this two counteracting effects leads to a sightly increase of the distance spent in the step climb phases for higher $\mathrm{ROC}_{\min }$.

A prominent result is observed for $90 \%$ of MLM and CI $45 \mathrm{~kg} \mathrm{~min}^{-1}$. In this case, reducing the $\mathrm{ROC}_{\text {min }}$ from $500 \mathrm{ft} \mathrm{min}^{-1}$ to $100 \mathrm{ft} \mathrm{min}^{-1}$ doubles the amount of distance not at constant altitude. This is because not only the number of step climbs per nautical mile are almost equal, but also the amount of time spent on each step climb quintuples.

According to Fig. 3, changing the minimum rate of climb from $500 \mathrm{ft} \mathrm{min}^{-1}$ to $300 \mathrm{ft} \mathrm{min}^{-1}$, the additional percentage of trip distance not performed at constant altitude would be lower than a $5 \%$ for the six combinations of CI and LM analysed in this paper, whilst the number of step climbs per nautical mile would be almost unaffected. These results combined with those obtained in the preceding Section suggest that a reduction of the $\mathrm{ROC}_{\min }$ to $300 \mathrm{ft} \min ^{-1}$ would lead to a interesting compromise between "enough" operating cost benefits and an impact on the ATM low "enough". 


\section{Effects of the step climbs height}

Another approach to bring conventional operations closer to continuous cruise climb might consist in reducing the step climbs height. It is expected that by performing shorter step climbs, but more often, relaxed cruise operations would enhance the efficiency of the cruise phase of flight. For this section two $\mathrm{ROC}_{\text {min }}$ values have been considered: $\mathrm{ROC}_{\text {min }}=500 \mathrm{ft} \mathrm{min}{ }^{-1}$ (corresponding to current regulations) and $\mathrm{ROC}_{\min }=300 \mathrm{ft} \mathrm{min}^{-1}$ (as found in previous section as potentially interesting for future ATM paradigms).

To illustrate the effects of the vertical separation between consecutive flight levels, Fig. 4 shows the vertical profiles above FL300, as a function of the distance to go, for different heights of the step climbs. Figs. 4(b) and 4 (a) correspond to the cases with $\mathrm{ROC}_{\min }$ of $500 \mathrm{ft} \mathrm{min}^{-1}$ and $300 \mathrm{ft} \mathrm{min}^{-1}$, respectively. In both cases, maximum range operations for a trip distance of $2800 \mathrm{NM}$ and a LM of $80 \%$ of the MLM have been considered.

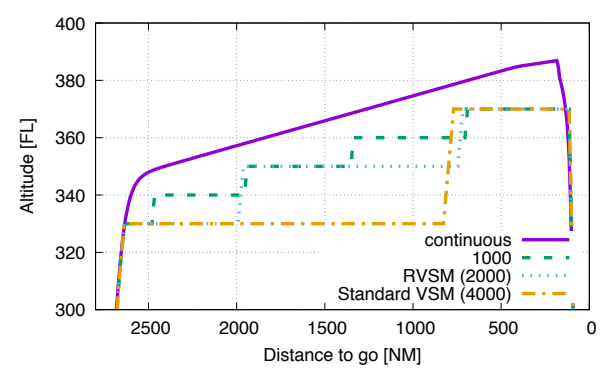

(a) $500 \mathrm{ft} \mathrm{min}^{-1}$

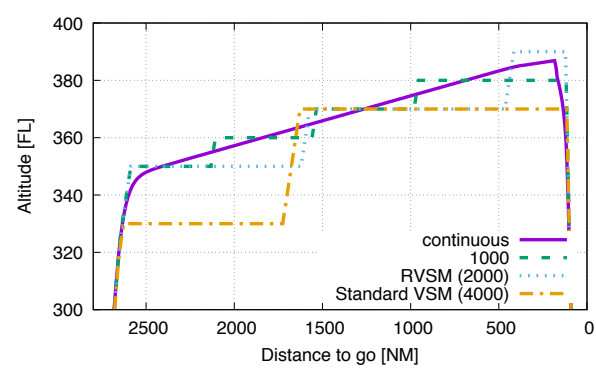

(b) $300 \mathrm{ft} \min ^{-1}$

Figure 4: Vertical profiles for different spacing between consecutive cruise levels

According to Fig. 4, the lower the step climbs height, the closer the trajectory to the unconstrained continuous profile and the larger the amount of cruise level changes. Consequently, less differences in terms of total operating cost are observed for lower heights of the step climbs at the expense of more step climbs per NM (see next section for a detailed analysis).

It is very interesting to note that by reducing the heights of the step climbs, whilst keeping constant the $\mathrm{ROC}_{\text {min }}$, the optimal initial and final cruise levels may also change (see for instance Fig. 4(b)).

\subsection{Impact on the operating costs}

Fig. 5 shows the relative operating cost differences between relaxed cruise operations and continuous cruise climb considering the different independent 
variables of Table 2. The extra cost of conventional operations in the standard VSM and RVSM airspaces are also shown in these Figures.
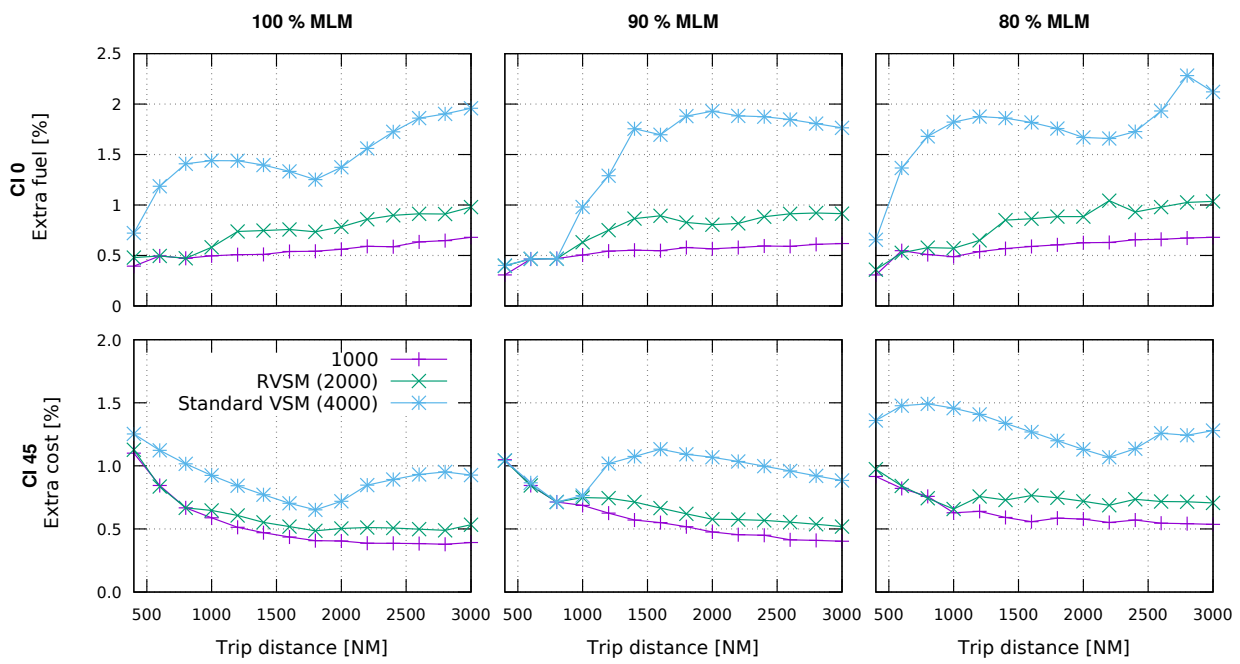

(a) $\mathrm{ROC}_{\min }=500 \mathrm{ft} \min ^{-1}$
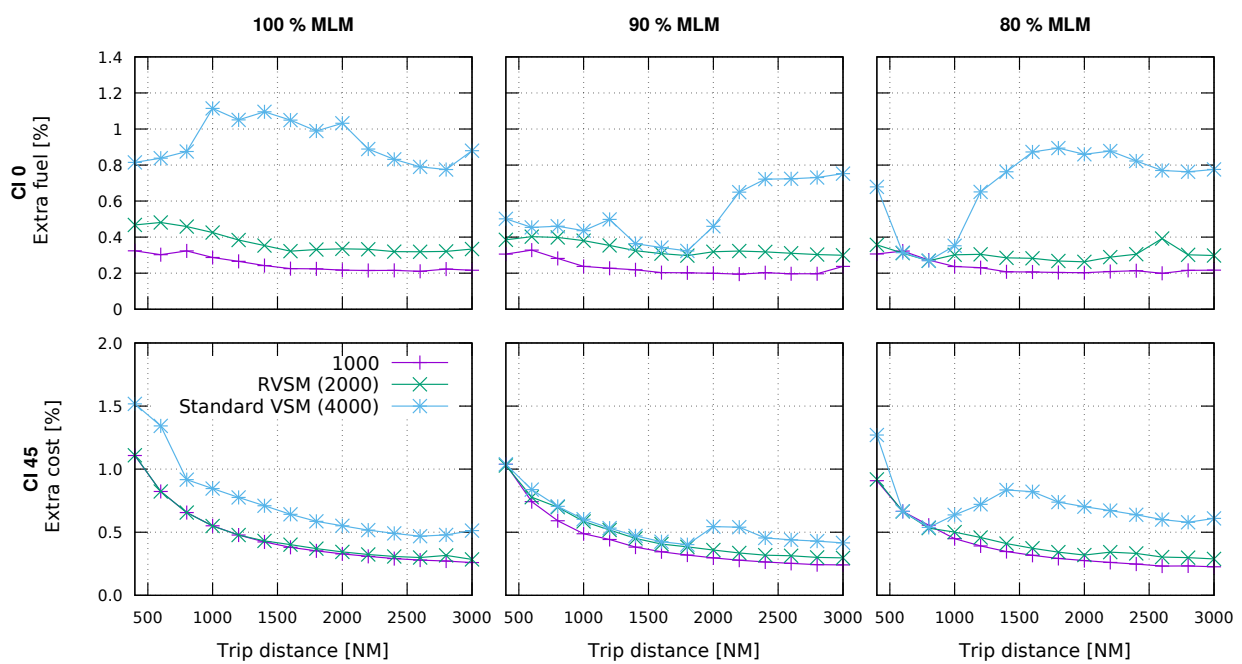

(b) $\mathrm{ROC}_{\min }=300 \mathrm{ft} \mathrm{min}^{-1}$

Figure 5: Relative operating cost differences with respect to continuous cruise climb for different spacing between cruise levels 
According to Fig. 5(a), by flying conventional operations $\left(\mathrm{ROC}_{\min }=\right.$ $500 \mathrm{ft} \mathrm{min}^{-1}$ ) in RVSM, the relative extra cost approximately halves if compared with VSM (from 0.5-2\% to 0.5-1\%). As expected, the introduction of relaxed cruise operations with a height of the step climbs of $1,000 \mathrm{ft}$ would reduce the relative extra cost even further (down to 0.5-0.6\%).

Regarding the effect of the CI on the relative extra cost, the same reasoning discussed in Section 3.1 applies here. In this case, however, the differences between RVSM airspace and the proposed spacing of $1,000 \mathrm{ft}$ for CI $45 \mathrm{~kg} \mathrm{~min}{ }^{-1}$ are not as significant as for maximum range operations.

As already shown in Section 3.1, the relative extra costs when flying with a less restrictive $\mathrm{ROC}_{\text {min }}$ are significantly reduced if compared with conventional operations. According to Fig. 5(b), for VSM airspace and maximum range operations, the maximum extra cost is reduced to $1 \%$ by using a $\mathrm{ROC}_{\text {min }}$ equal to $300 \mathrm{ft} \mathrm{min}{ }^{-1}$. For this relaxed $\mathrm{ROC}_{\text {min }}$, the difference between the proposed solution $(1,000 \mathrm{ft})$ and the RVSM airspace is about $0.1 \%$ for CI 0 and even less for operations with CI $45 \mathrm{~kg} \mathrm{~min}^{-1}$.

These results manifest that reducing the height of cruise step climbs to $1,000 \mathrm{ft}$, whilst keeping the suggested minimum rate of climb of $300 \mathrm{ft} \mathrm{min}^{-1}$ would not provide significant cost benefits if compared with the current flight orientation schemes in RVSM airspace.

\subsection{Impact on the ATM}

Although relaxed cruise operations with reduced height of the step climbs could lead to benefits regarding flight efficiency, it is questionable if this reduction in vertical separation between traffic flows could negatively impact the safety of the operations. This study, however, is out of the scope of this paper. In this Section, the impact on the ATM is analysed by considering the same metrics as in Section 3.2.

Figures 6 and 7 show the impact on the ATM considering a $\mathrm{ROC}_{\text {min }}$ of $500 \mathrm{ft} \min ^{-1}$ and $300 \mathrm{ft} \mathrm{min}^{-1}$, respectively.

According to Figs. 6 and 7 the number of step climbs per NM with a height of the step climbs of 1,000 ft significantly increases if compared with RVSM and VSM standard airspaces (regardless of the $\mathrm{ROC}_{\text {min }}$ ). Interestingly, the percentage of the total trip distance which is not performed at constant altitude differs by less than $3 \%$ for all the combinations of CI and LM considered in this paper. For those simulations in which the initial and final cruise levels remain unmodified regardless of the separation between consecutive cruise levels (e.g. Fig. 4(a)), the climb and descent distances 

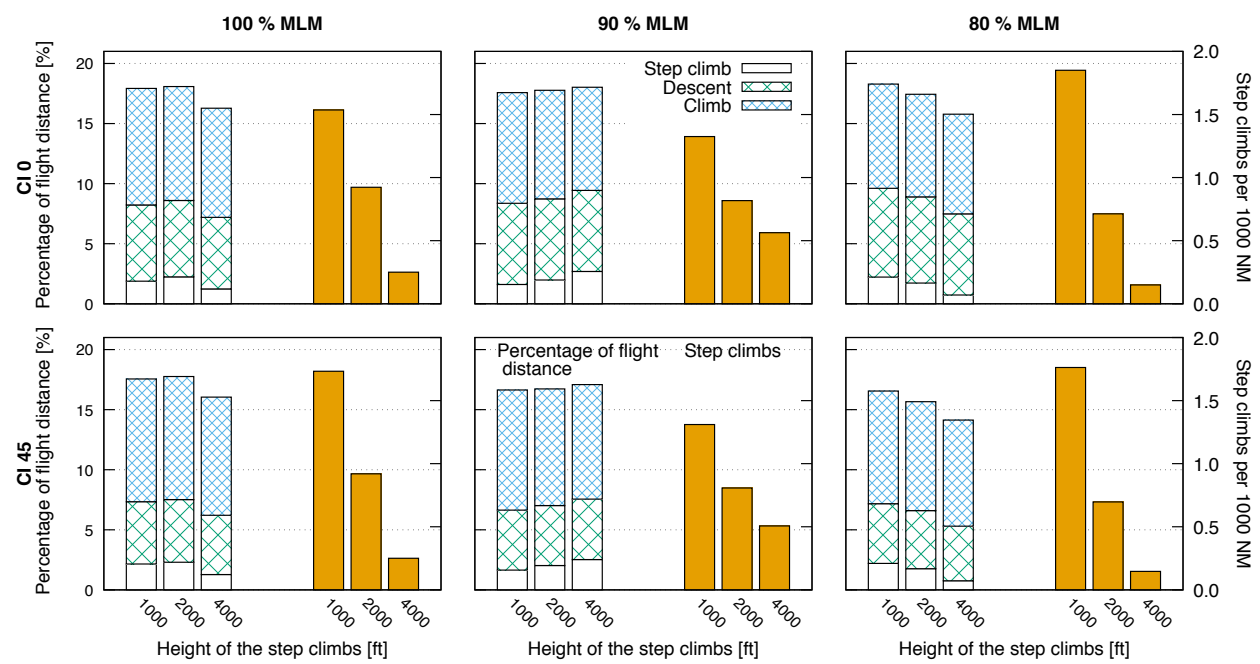

Figure 6: Impact on the ATM for different spacing between cruise levels at $\mathrm{ROC}_{\min }=$ $500 \mathrm{ft} \min ^{-} 1$
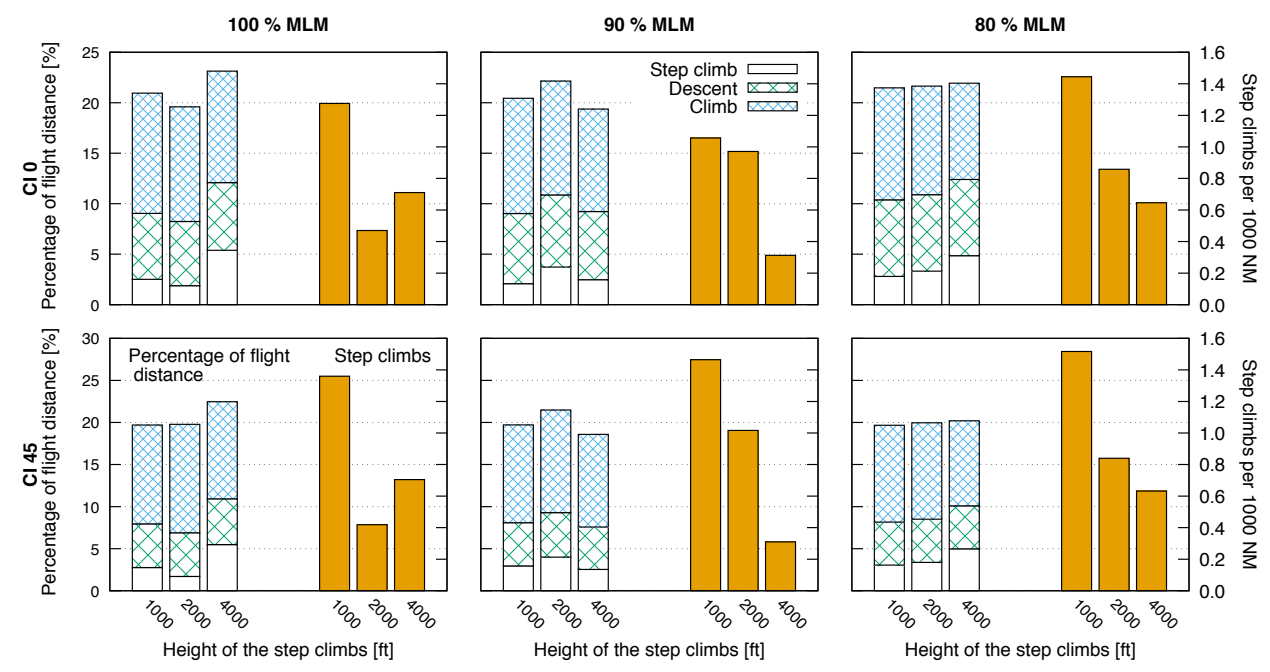

Figure 7: Impact on the ATM for different spacing between cruise levels at $\mathrm{ROC}_{\min }=$ $300 \mathrm{ft} \min ^{-} 1$

keep constant too. Then, although the number of step climbs increases by reducing the spacing between cruise levels, the time (and distance) needed 
to perform a step climb decreases. The combination of these two counteracting behaviours lead to an insignificant change of the total trip distance not performed at constant altitude.

Of particular interest are the results obtained for $100 \%$ of the MLM con-

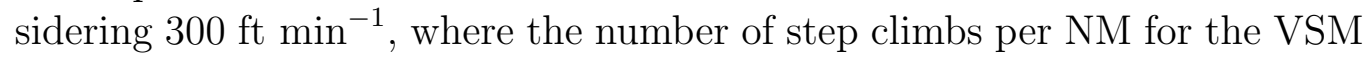
airspace is higher than for the RVSM airspace. For the scenarios in which VSM airspace are considered, a step climb from FL330 to FL370 is performed 400 nautical miles before the top of descent. Instead, the last cruise level change in RVSM airspace is performed 1500 NM before the TOD. This implies that for those simulations in between 400 and $1500 \mathrm{NM}$ the number of step climbs is one for VSM and zero for RVSM, affecting the mean value as observed in Fig. 7.

\section{Conclusions}

The reduction of the environmental impact of air transportation, without compromising safety and airspace capacity, is perhaps one of the major concerns of all aviation stakeholders. Future air traffic management (ATM) enhancements, as planned in SESAR and NextGen programs, should contribute to reduce fuel burnt (and consequently gaseous emissions), and to improve on operational safety and ATM cost-efficiency. Even small amounts of cost savings become significant at aggregate level, especially when considering the high volume of traffic that is operating every day. This paper has focused on the potential savings of the introduction of eventual relaxed cruise operations, showing already some noteworthy figures in terms of total operating cost.

The extra cost associated with optimal conventional operations ranges from $0.5 \%$ to $1 \%$ if compared to continuous cruise climb in cruise. Results showed that by reducing the minimum rate of climb to $300 \mathrm{ft} \min ^{-1}$, in a Reduced Vertical Separation Minima airspace (RVSM), the extra cost would reduce a $0.3 \%$ approximately. This reduction on the operating cost would be accompanied by an increment around $5 \%$ of the percentage of flight distance not performed at constant altitude.

Reducing the height of the step climbs to 1,000 ft, whilst keeping the current minimum rate of climb regulation at $500 \mathrm{ft} \min ^{-1}$, would reduce the extra cost below $0.5-0.6 \%$. However, the number of step climbs per nautical mile would double, impacting in this way on air traffic control (ATC) workload. 
Conclusions arising from this paper suggest to reduce the minimum rate of climb to $300 \mathrm{ft} \mathrm{min}^{-1}$ whilst keeping the RVSM standard. This approach gives a good compromise between cost savings and impact on the ATM. A further reduction on the minimum rate of climb and/or the height of the step climbs would not entail significant cost savings and would suppose a large negative effect on the ATM.

It is expected that in a near future, relaxed cruise operations would take advantage of advanced aircraft surveillance applications (e.g. Airborne Separation Assurance Systems, ASAS) [22] using new aircraft surveillance systems (e.g. Automatic Dependent Surveillance-Broadcast, ADS-B) to reduce the vertical speed and/or the spacing between cruise levels, aiming at exploiting the evident benefits that fully continuous cruise climb entail.

These results evidenced that a more cost-efficient and environmentally friendly air transport system could be achieved if the ATM practices were adapted to guarantee safety and capacity for such a new types of operations.

[1] H. Erzberger, J. D. Mclean, J. F. Barman, Fixed-range optimum trajectories for short-haul aircraft, Tech. Rep. D-8115, NASA, Washington, D.C. (Dec. 1975).

[2] E. L. Miller, Optimal cruise performance, Journal of Aircraft 30 (3) (1993) 403-405.

[3] R. Dalmau, X. Prats, Fuel and time savings by flying continuous cruise climbs: Estimating the benefit pools for maximum range operations, Transportation Research - Part D: Transport and Environment 35 (2015) 62-71. doi:http://dx.doi.org/10.1016/j.trd.2014.11.019.

[4] H. G. Visser, S. Hartjes, Economic and environmental optimization of flight trajectories connecting a city-pair, Proceedings of the Institution of Mechanical Engineers, Part G: Journal of Aerospace Engineering 228 (6) (2014) 980-993. doi:10.1177/0954410013485348.

[5] R. Dalmau, X. Prats, How much fuel and time can be saved in a perfect flight trajectory? continuous cruise climbs vs. conventional operations, in: Proceedings of the 6th International Congress on Research in Air Transportation (ICRAT), Eurocontrol/FAA, Istanbul, Turkey, 2014, best paper in track award. 
[6] J. A. Lovegren, R. J. Hansman, Estimation of potential aircraft fuel burn reduction in cruise via speed and altitude optimization strategies, Tech. rep., MIT International Center for Air Transport (ICAT), Cambridge, USA (Feb. 2011).

[7] C. Nutt, Nats fuel efficiency metric, Tech. Rep OA-1161 Version 1.3, National Air Traffic Services (NATS) (2012).

[8] SESAR Joint Undertaking, Reduction of emissions on the north atlantic by the implementation of ads-b, Tech. Rep SJU/0002-CFP (2010).

[9] K. B. Marais, T. G. Reynolds, P. Uday, D. Muller, J. Lovegren, J.-M. Dumont, R. J. Hansman, Evaluation of potential near-term operational changes to mitigate environmental impacts of aviation, Proceedings of the Institution of Mechanical Engineers, Part G: Journal of Aerospace Engineering 227 (8) (2013) 1277-1299. doi:10.1177/0954410012454095.

[10] S. Ruiz, M. Soler, Conflict pattern analysis under the consideration of optimal trajectories in the european atm, in: Proceedings of the 11th USA/Europe Air Traffic Management Research and Development Seminar (ATM2015), Eurocontrol/FAA, Lisbon, Portugal, 2015.

[11] International Civil Aviation Organization (ICAO), Manual on Implementation of a $300 \mathrm{~m}$ (1 $000 \mathrm{ft})$ Vertical Separation Minimum Between FL 290 and FL 410 Inclusive (Doc 9574 AN/934), 2nd Edition (2002).

[12] International Civil Aviation Organization (ICAO), Procedures for Air Navigation Services: Air Traffic Management (Doc 4444 ATM/501), 15th Edition (2007).

[13] U.S. Dept. of Transportation, Federal Aviation Administration (F.A.A), Aeronautical Information Publication Manual (AIMP): Chapter 4 - Subchapter F - Section 4 - Part 1091 (2014).

[14] Great Britain Civil Aviation Authority, Aeronautical information publication (AIP) United Kingdom: En-route (ENR) 1.1 - general rules (2013).

[15] J. Hoekstra, Aircraft performance models for atm research, in: Tutorials at the 7th International Congress on Research in Air Transportation (ICRAT), FAA/EUROCONTROL, Philadelphia (EEUU), 2016. 
[16] A. E. Bryson, Y. Ho, Applied optimal control: optimization, estimation, and control, Taylor and Francis Group, New York, USA, 1975.

[17] J. T. Betts, Practical Methods for Optimal Control and Estimation Using Nonlinear Programming, 2nd Edition, SIAM, 2010. doi: $10.1137 / 1.9780898718577$.

[18] J. T. Betts, Survey of Numerical Methods for Trajectory Optimization, Journal of Guidance, Control, and Dynamics 21 (2) (1998) 193-207. doi:10.2514/2.4231.

[19] Airbus, Flight Crew Operation Manual (FCOM). A320. Version 1.3.1, Training and flight operations support and services, Toulouse, France (1993).

[20] Airbus, Getting to grips with the cost index, Flight Operations Support and Line Assistance, Blagnac, France (May 1998).

[21] W. Roberson, R. Root, D. Adams, Fuel Conservation Strategies: cruise flight, Boeing Aero Quaterly, The Boeing Company (2008).

[22] CARE/ASAS, Co-operative actions of r\&d in eurocontrol action on airborne separation assistance system, Tech. rep., EUROCONTROL (november 2004). 